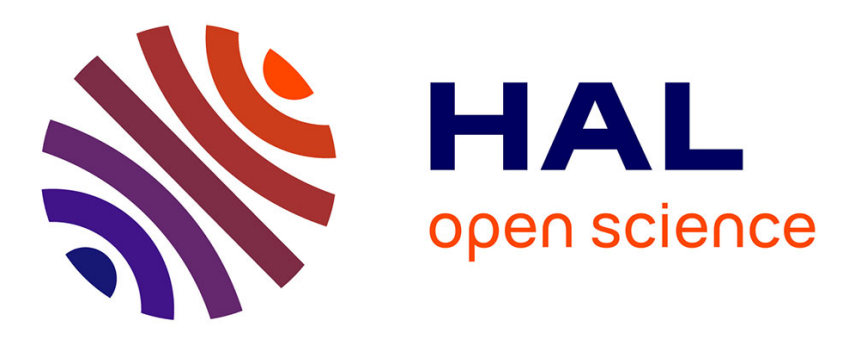

\title{
Effect of turbulence on the wavefront of an ultra high intensity laser beam
}

Morgane Bellec, Alain Girard, Guillaume Balarac, Ulrich Bieder, François Millet, N. Luchier

\section{- To cite this version:}

Morgane Bellec, Alain Girard, Guillaume Balarac, Ulrich Bieder, François Millet, et al.. Effect of turbulence on the wavefront of an ultra high intensity laser beam. Journal of Fluids Engineering, 2020, 10.1115/1.4049113 . hal-02572291v1

\section{HAL Id: hal-02572291 \\ https://hal.science/hal-02572291v1}

Submitted on 13 May 2020 (v1), last revised 30 Nov 2020 (v2)

HAL is a multi-disciplinary open access archive for the deposit and dissemination of scientific research documents, whether they are published or not. The documents may come from teaching and research institutions in France or abroad, or from public or private research centers.
L'archive ouverte pluridisciplinaire HAL, est destinée au dépôt et à la diffusion de documents scientifiques de niveau recherche, publiés ou non, émanant des établissements d'enseignement et de recherche français ou étrangers, des laboratoires publics ou privés. 


\section{Effect of turbulence on the wavefront of a ultra high intensity laser beam}

\author{
Morgane Bellec \\ Univ. Grenoble Alpes \\ CEA, IRIG, DSBT \\ Grenoble, France \\ Email: morgane.bellec@gresille.org
}

\author{
Guillaume Balarac \\ Univ. Grenoble Alpes \\ CNRS, LEGI, Grenoble, France \\ Institut Universitaire de France (IUF)
}

\author{
François Millet \\ Univ. Grenoble Alpes \\ CEA, IRIG, DSBT \\ Grenoble, France
}

\author{
Alain Girard \\ Univ. Grenoble Alpes \\ CEA, IRIG, DSBT \\ Grenoble, France \\ Email: alain.girard@cea.fr
}

\author{
Ulrich Bieder \\ CEA, DEN, STMF \\ Université Paris-Saclay \\ Gif-sur-Yvette, France
}

\author{
Nicolas Luchier \\ Univ. Grenoble Alpes \\ CEA, IRIG, DSBT \\ Grenoble, France
}

Ultra high intensity lasers face thermal management issues that limit their repetition rates. The key challenge is to effciently evacuate the heat deposited in the amplifier by the optical pumping without impacting the output laser beam quality. The amplifier can have a multi-slab geometry where the laser beam crosses successive amplifying slabs and the cooling channels that separate them. The present work investigates numerically how a cryogenic cooling of the multi-slab amplifier by turbulent channel flows may affect the wavefront of the laser beam. To this end, Large Eddy Simulations representative of the amplifier cooling are performed using Tri$o C F D$, a code developed by the CEA. First, validation simulations are carried out for heated channel flows, allowing comparisons to Direct Numerical Simulation results from the literature. Then Large Eddy Simulations of an open turbulent channel flow cooling two slabs are conducted using conjugated heat transfer between the solid and the fluid. The phase distortions, mean and fluctuations, induced by the inhomogeneous and turbulent temperature field are computed directly from the LES. The Sutton model is widely used in aero-optic studies; its validity and applicability to this problem is then examined. Finally, the effect of an increased optical heating of the slabs is investigated. It is the first time that TrioCFD is used to address the question of the impact of the cooling of laser amplifiers, and it has proven to be a valuable tool for this application.

\section{Introduction}

There is nowadays in Europe a strong interest in the field of high power lasers. Several multi-petawatt lasers are currently in operation, among which the three ELI projects in eastern Europe [1] and Apollon in France [2]. When designing the solid-state amplifier of a high power laser, thermal management is a critical element to attain a high repetition rate [3]. The solid-state laser amplifier DiPOLE100 (105 J per pulse) demonstrated stable operation at a repetition rate of $10 \mathrm{~Hz}$ using cryogenically cooled multi-slab amplifiers [4]. Cooling the amplifying material at cryogenic temperatures, i.e. below $150 \mathrm{~K}$, presents advantages both from the thermal and the optical point of view, as reviewed by Brown et al. [5]. Compared to room temperature, the thermal conductivity of amplifying materials is higher at low temperature. A more homogeneous temperature is therefore expected in the amplifying material, reducing the thermo-mechanical stresses and the geometric deformations. The optical performances are also enhanced, especially for ytterbium-doped crystals: from quasi-three level laser materials at $300 \mathrm{~K}$ they become four level materials at $77 \mathrm{~K}$, thus increasing the overall laser efficiency [6].

In a cryogenic multi-slab amplifier, the amplifying material is split into several slabs, which allows to increase the heat exchange surface [7]. The slabs are separated by cooling channel flows of cryogenic gaseous helium. In this 
configuration, the laser beam goes perpendicularly through the channel flows. The homogeneity of the refractive index in the media crossed by the laser beam is crucial to ensure the output beam coherence. As stated by the Gladstone-Dale law [8], the refractive index of a fluid varies with its density and thus with its temperature. Helium has the desired optical properties for this application: its thermo-optic coefficient at $100 \mathrm{~K}$ is $(d n / d T)_{f}=4.6 \times 10^{-6} \mathrm{~K}^{-1}$ [9]. By comparison, the thermo-optic coefficient of $1 \%$-doped Yb:YAG (ytterbium-doped crystal of yttrium aluminum garnet) at $100 \mathrm{~K}$ and $\lambda=1034 \mathrm{~nm}$ is $(d n / d T)_{s}=10^{-6}$ $\mathrm{K}^{-1}$ [10]. Due to the low kinematic viscosity of cryogenic helium $\left(v_{f}=3.10^{-6} \mathrm{~m}^{2} / \mathrm{s}\right.$ at $80 \mathrm{~K}$ and 5 bar [9]), the cooling channel flows will be turbulent at the mass flow rates needed to evacuate the heating in the amplifier. The velocity fluctuations induce temperature fluctuations in the fluid. The consequent refractive index inhomogeneities, however small given the fluid thermo-optic coefficient, may not be negligible. Their impact on the laser beam quality must be assessed.

Light propagation through a turbulent medium has first been studied by astronomers: the atmospheric turbulence usually degrades the resolution of a telescope [11]. Various techniques, including adaptive optics, were developed to mitigate those effects. Turbulence also affects the performance of airborne laser systems [12]. In this case the flow is compressible. The laser beam propagates through a medium with an inhomogeneous refractive index because of the density variations. Different parts of its wavefront travel at different speeds, causing wavefront aberrations. To predict the wavefront aberrations, Sutton [13] developed a model linking the wavefront phase distortion with the variance and the correlation length of the fluctuating density. One of the assumptions made is that the turbulent flow is homogeneous and isotropic. This assumption is met neither in the airborne applications where the considered flow is a compressible boundary layer, nor in the case of the cooling channels with developing thermal boundary layers. Tromeur et al. [14, 15] tested the Sutton model in a turbulent boundary layer flow by comparing it to Large Eddy Simulations (LES) and to experimental results. They recommend the use of LES to correctly capture the aero-optic effects as they found that the Sutton model was leading to significantly different wavefront aberrations compared to experiments. However, Wang and Wang [16] questioned Tromeur et al.'s formulation of the Sutton model, pointing this as the cause for their unsatisfactory results. They found good agreement between the Sutton model predictions and the directly integrated LES results by defining appropriately the density length scale. Truman and Lee [17] studied the propagation of an optical beam through a uniform shear turbulent flow without wall boundary. They used the instantaneous passive-scalar field results obtained by Direct Numerical Simulation (DNS) of such a flow to represent the fluctuating refractive index field. They found a significant phase distortion of the optical beam that can be minimized by adjusting the angle of the beam so that the direction of propagation is approximately normal to the turbulent structures. To the best of the authors'

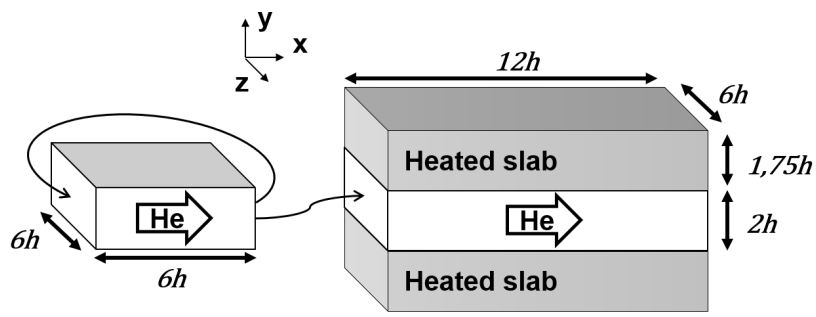

Fig. 1. Computational domains with precursor flow channel, open flow channel and heated solid slabs. $h=0.002 \mathrm{~m}$.

knowledge, there exists no such study in an actual wallbounded flow with developing thermal boundary layers. Most aero-optics studies of flows with heat exchange are experimental [18]. The closest case was conducted again by Truman [19] in a periodic turbulent channel flow at low Reynolds number $\left(R e_{\tau}=180\right)$ by DNS. He showed that the phase distortion is mainly due to the large scale turbulent structures of the flow. Filtering out the small scale fluctuations (arbitrarily chosen in his case as smaller than one root mean square) permitted to retrieve $85 \%$ of the phase distortion. This advocates for the usage of LES in aero-optics studies.

The aim of this work is to analyze the effect of the turbulent gaseous cooling of a laser amplifier on the wavefront of the laser beam using TrioCFD, a code developed by the CEA [20]. The mean phase distortion could be compensated by adaptive optics, but not the fluctuations. It is therefore crucial to assess their impact on the laser beam quality in order to provide some guidance for future design and operation. As the statistical approach (i.e. Reynolds-Averaged Navier-Stokes simulations) is inadequate to investigate the phase distortion fluctuations, Large Eddy Simulations are carried out. To represent the cryogenic cooling of a high power and high repetition rate laser, a plane turbulent open channel flow is first simulated. The fluid, gaseous helium at $80 \mathrm{~K}$, cools two solid slabs made of Yb:YAG amplifying crystal. The resulting temperature distribution is analyzed from the optical point of view. The paper is organized as follows: the section 2 describes the numerical methods, both for the thermo-hydraulics and the optics aspects; the section 2.2 assesses the numerical model by comparing dynamical and thermal profiles to the literature; the section 3.1 presents the temperature field in the amplifier and the section 3.2 investigates its effect on the laser beam; finally the section 3.3 deals with the consequences of an increased optical heating of the slabs.

\section{Numerical methods \\ 2.1 Large Eddy Simulation}

The purpose of this work is to investigate the cryogenic cooling of amplifiers by a gaseous helium flow for high power lasers. The characteristics of the studied case were chosen so as to be as representative as possible of an actual multi-slab amplifier, while accounting for computational 
Table 1. Physical properties of the amplifying material and of the gaseous helium at $80 \mathrm{~K}$ and 5 bar

\begin{tabular}{lll}
\hline & Fluid & Solid \\
\hline Kinematic viscosity $v\left(\mathrm{~m}^{2} / \mathrm{s}\right)$ & $3.10^{-6}$ & - \\
Thermal conductivity $\lambda(\mathrm{W} / \mathrm{mK})$ & 0.064 & 35 \\
Heat capacity $C_{p}(\mathrm{~J} / \mathrm{kgK})$ & 5205 & 100 \\
Density $\rho\left(\mathrm{kg} / \mathrm{m}^{3}\right)$ & 2.982 & 4583 \\
\hline
\end{tabular}

cost considerations. Figure 1 shows a schematic view of the computational domains. Only a portion of one of the cooling channels is simulated. The dimensions of the main channel are $0.024 \mathrm{~m} \times 0.004 \mathrm{~m} \times 0.012 \mathrm{~m}$. The two solid plates have the same length and width, and they are $0.0035 \mathrm{~m}$ thick. An actual slab would typically be wider streamwise and spanwise (about $0.1 \mathrm{~m} \times 0.1 \mathrm{~m}$ [21]), but the crosswise dimensions are respected. The flow is assumed to be fully developed, with a bulk velocity of $10 \mathrm{~m} / \mathrm{s}$. That corresponds to a bulk Reynolds number of $R e_{b}=14000$. To generate the velocity inlet, a classical technique (see for example [22]) is used: a so-called precursor channel is simulated. It is an isothermal bi-periodic channel whose spanwise and crosswise dimensions are the same as the main channel ones, but with half the length.

The properties of the fluid and the solid are summarized in Tab. 1. They are conventional values for a cryogenically cooled amplifier. The fluid is helium at $80 \mathrm{~K}$ and 5 bar, whose properties are taken from the database Hepak [9]. The properties are taken constant and the flow is considered incompressible. The solid properties are given for 1\%-doped Yb:YAG at $80 \mathrm{~K}$, as measured by Cardinali [10]. A bulk heating of $9.2 \mathrm{~W}$ is deposited homogeneously in the two solid volumes. The fluid evacuates it via conjugate heat transfer with the solid. This heat deposit is proportional in volume to a typical heat deposit in a laser amplifier [23]. In an actual amplifier however, the heat is deposited at discrete times corresponding to the pumping pulses. Typical pulses last a few nanoseconds, and they have a repetition rate of about $10 \mathrm{~Hz}$. A thermal balance calculation shows that the solid temperature decreases by about $1 \mathrm{~K}$, i.e. about $1 \%$, between each pulse at thermal equilibrium. In this work, the heating is constant in time, and the results presented are taken at thermal equilibrium. This is justified below by the different time scales encountered.

The governing equations are the incompressible NavierStokes equations for the fluid, and the energy conservation equations for the fluid and the solid. In Large Eddy Simulation, or LES, (see e.g. [22]) the large scales of turbulence are resolved while the effect of the small scales (smaller than the grid characteristic size) is modeled. The sub-grid scale model used in this work is the wall-adapting local eddyviscosity (WALE) model of Nicoud and Ducros [24]. The governing equations are written below, where $\sim$ denotes the
LES filtering.

$$
\left\{\begin{array}{l}
\frac{\partial \tilde{u_{i}}}{\partial x_{i}}=0 \\
\frac{\partial \tilde{u_{i}}}{\partial t}+\frac{\partial \tilde{u_{i}} \tilde{u_{j}}}{\partial x_{j}}=-\frac{1}{\rho_{f}} \frac{\partial \tilde{P}}{\partial x_{i}}+v \frac{\partial}{\partial x_{j}}\left(\frac{\partial \tilde{u_{i}}}{\partial x_{j}}+\frac{\partial \tilde{u_{j}}}{\partial x_{i}}\right)-\frac{\partial \tau_{i j}}{\partial x_{j}} \\
\frac{\partial \tilde{T}}{\partial t}+\frac{\partial \tilde{u_{i}} \tilde{T}}{\partial x_{i}}=\alpha_{f} \frac{\partial^{2} \tilde{T}}{\partial x_{i}^{2}}-\frac{\partial \theta_{i}}{\partial x_{i}} \\
\frac{\partial T}{\partial t}=\alpha_{s} \frac{\partial^{2} T}{\partial x_{i}^{2}}+\frac{1}{C_{p s} \rho_{s}} Q
\end{array}\right.
$$

Here, $u_{i}$ are the velocity components in the $x_{i}$ directions, $P$ is the pressure, $T$ is the temperature. The material properties - the kinematic viscosity $v$, the density $\rho$, the thermal diffusivity $\alpha$ and the heat capacity $C_{p}$ - have the subscript $s$ or $f$ referring to solid or fluid respectively. $Q$ is the volumetric heat deposit. Finally, $\tau_{i j}=\tilde{u_{i}} \tilde{u_{j}}-\tilde{u_{i}} \tilde{u_{j}}$ and $\theta_{i}=\tilde{u_{i} T}-\tilde{u_{i} T}$ are respectively the sub-grid scale stress tensor and the subgrid scale temperature flux to model in LES.

The simulations were performed using TrioCFD [20], an open-source Computational Fluid Dynamics code supported by the CEA, formerly known as Trio_U [25]. The spatial discretization used in this work is a "Finite DifferenceVolume" method for hexahedral structured grid where the scalar unknowns are computed at the center of the cells while the velocities are computed at the center of the faces. The mesh comprises $60 \times 192 \times 240$ cells for the precursor channel and $120 \times 192 \times 240$ cells for the main open channel. With a Reynolds number based on the friction velocity (see Eqn. (3)) of $R e_{\tau}=400$, the streamwise and spanwise dimensionless sizes are respectively $\Delta x^{+}=40$ and $\Delta z^{+}=10$. The crosswise dimensionless size varies between $\Delta y^{+}=1$ at the wall and $\Delta y^{+}=15$ in the center of the channel, following a tangential hyperbolic growing law. These sizes are typical resolutions for a LES, which is thus expected to correctly capture the aero-optical effects according to Mani et. al. analysis of grid requirements [26]. Each solid plate mesh consists of $120 \times 30 \times 240$ cells homogeneously distributed. Time marching is explicit and relies on a third order RungeKutta scheme. Second order centered schemes are used for the energy and momentum diffusion, as well as the dynamic convection. The thermal convection uses a third order QUICK scheme (Quadratic Upstream Interpolation for Convective Kinematics). The time step varies between $7 \times 10^{-7} \mathrm{~s}$ and $9 \times 10^{-7} \mathrm{~s}$, it is chosen so as to ensure the stability condition: Courant number of 1 . The simulations were run on the HPC resources of CINES, and 252 cores were used in parallel.

A major challenge presented by this simulation comes from the different characteristic times of the phenomena investigated: the solid conduction is slow compared to the turbulent convection. A heat balance leads to a thermal characteristic 


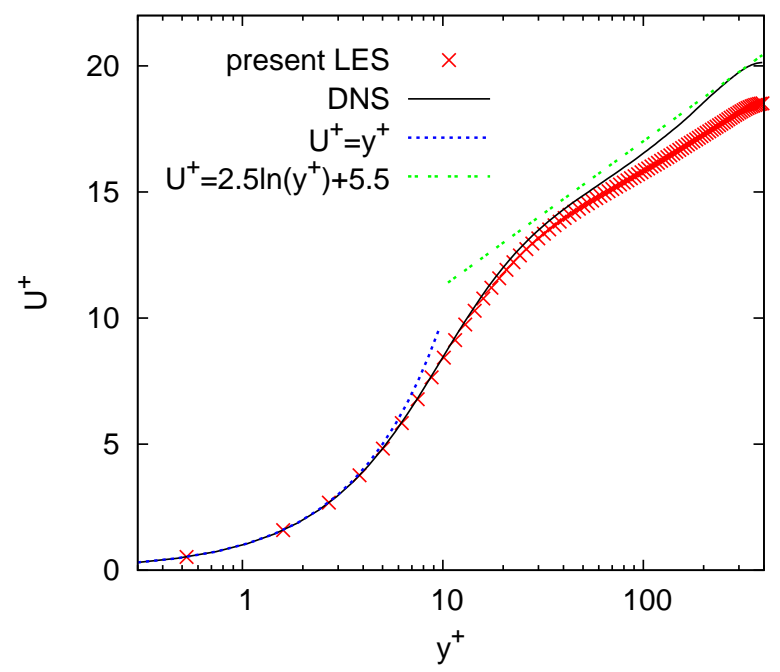

Fig. 2. Mean longitudinal velocity standardized by the friction velocity and compared to the DNS results of Moser et al. [28]

time of $\tau_{t h}=\frac{\rho_{s} C_{p s} L_{s}}{h_{\text {exch }}}=2.6 \mathrm{~s}$, where $L_{s}$ is the solid plate thickness, and $h_{\text {exch }}$ is the exchange coefficient evaluated by the classical Dittus-Boelter correlation [27]. This long time constant of the amplifier with respect to the repetition time $(0.1 \mathrm{~s})$ justifies the assumption of a constant heating. By comparison, the turbulence characteristic time is of three orders of magnitude smaller: $\tau_{f l}=\frac{h}{u_{\tau}}=3.5 \times 10^{-3} \mathrm{~s}$ where $h$ is the channel half-height and $u_{\tau}$ is the friction velocity defined Eqn. (3)). The computational cost of attaining the thermal equilibrium while correctly capturing the turbulent flow was decreased in different ways. First, the initial condition should be as close as possible to the thermal equilibrium solution. A preliminary calculation using RANS model was performed to provide this initial condition. Then the thermal characteristic time was artificially decreased by lowering the value of the heat capacity $C_{p s}$ by a factor of ten during the transient state. This adjustment is limited as a smaller heat capacity of the solid leads to smaller time steps for the solid conduction equation. The transient state was thus lowered to about 1 physical second. The statistics were then collected for more than 0.4 physical seconds, which corresponds to 120 turbulence characteristic times or 160 mean channel crossings. This average over time is denoted by \langle\rangle .

\subsection{Model validation}

In the first place, the numerical choices are assessed by comparisons with the literature in cases as close as possible to ours. Figures 2 and 3 present respectively the mean longitudinal velocity and the velocity root mean squares. The results are compared to Moser et al. [28] DNS results. They are presented in their normalized form, using the classical scaling [22].

$$
y^{+}=\frac{y u_{\tau}}{v} \quad U_{i}^{+}=\frac{\langle u\rangle}{u_{\tau}} \quad u_{i_{r m s}}^{+}=\frac{u_{i_{r m s}}}{u_{\tau}}
$$

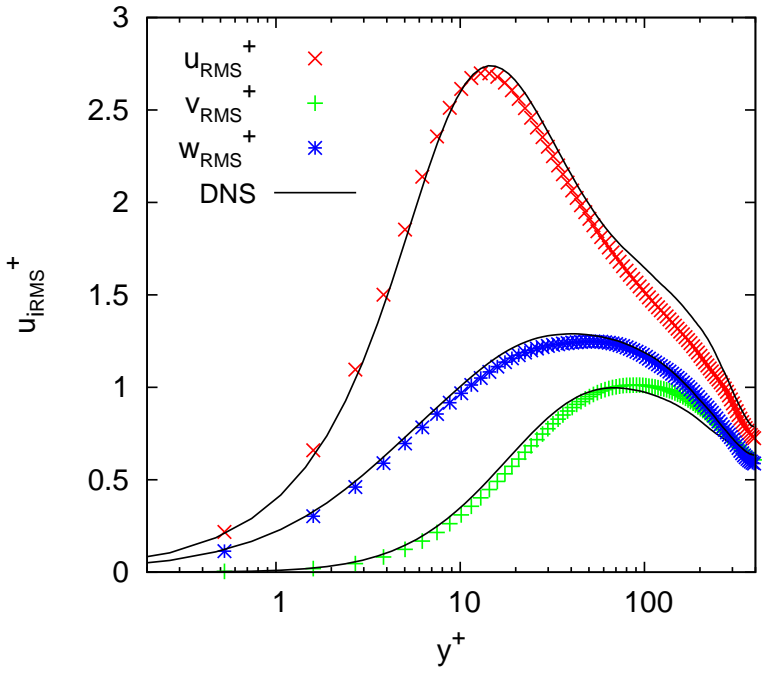

Fig. 3. Velocity root mean squares standardized by the friction velocity and compared to the DNS results of Moser et al. [28]

with $u_{\tau}$ is the friction velocity, defined by (see e.g. [29])

$$
u_{\tau}=\sqrt{v\left(\frac{d\langle u\rangle}{d y}\right)_{w}},
$$

where the subscript $w$ denotes the wall ( $y=0$ or $y=2 h$ ). The mean longitudinal velocity profile, plotted Fig. 2, shows a good agreement between the present LES and the reference DNS in the viscous sub-layer close to the wall. The standardized mean velocity is however under-estimated in the center of the channel by $10 \%$ at most. The standardized velocity root mean squares are nevertheless well captured, as can be seen from the satisfying agreement with DNS in Fig. 3. The velocity results could be improved by adopting a higher order scheme, at the expense of a higher numerical cost. It seems however unnecessary as the mean velocity under-estimation appears to have little impact on the mean temperature (see Fig. 4 discussed below).

In the second place, the temperatures are also assessed by comparison with the literature. To this end, the calculation has been conducted in a case similar to the one presented in section 2 except that the channel was here streamwise periodic. The precursor channel was therefore not needed, and the outlet velocity and temperature fields were used as inlet boundary conditions. All the numerical and physical characteristics of the plates and of the fluid were unchanged. A negative volumetric thermal source term was added in the fluid in order to exactly compensate the heat deposit in the plates so that the case would reach thermal equilibrium. Figures 4 and 5 present respectively the standardized mean temperature and the temperature root mean square. The results are compared to Kawamura et al. [30] DNS results. The classi- 


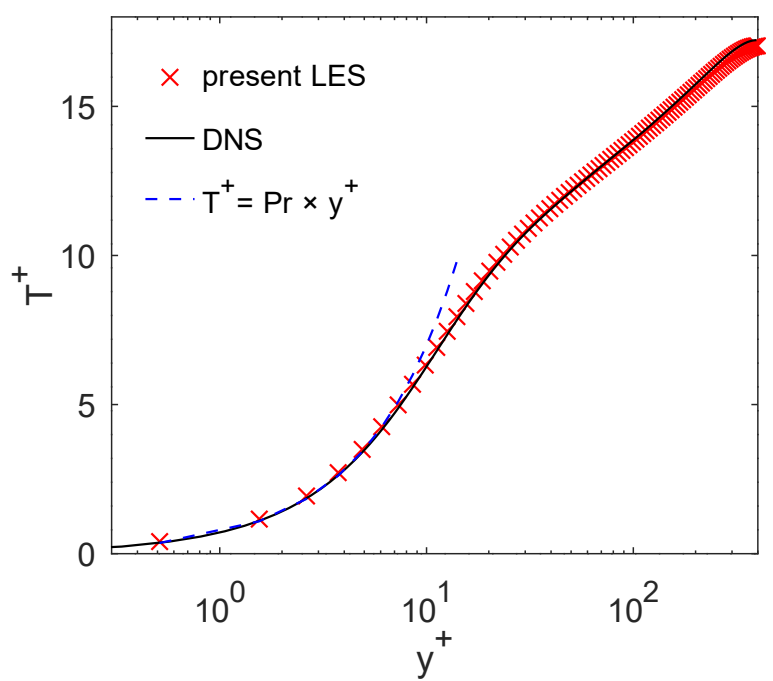

Fig. 4. Mean temperature standardized by the friction temperature and compared to the DNS results of Kawamura et al. [30]

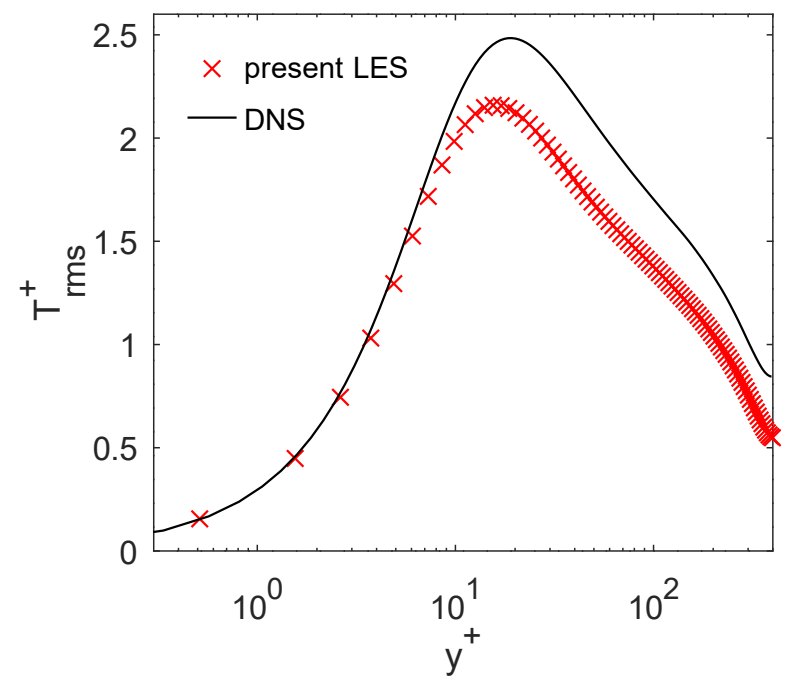

Fig. 5. Temperature root mean square standardized by the friction temperature and compared to the DNS results of Kawamura et al. [30]

cal scaling [22] used is

$$
T^{+}=\frac{T_{w}-\langle T\rangle}{T_{\tau}} \quad T_{r m s}^{+}=\frac{T_{r m s}}{T_{\tau}}
$$

with $T_{w}$ the wall temperature and $T_{\tau}$ the friction temperature, calculated from the wall normal gradient of the mean temperature and the friction velocity.

$$
T_{\tau}=\frac{\lambda\left(\frac{d\langle T\rangle}{d y}\right)_{w}}{\rho C_{p} u_{\tau}}
$$

The mean temperature profile plotted on Fig. 4 shows an ex- cellent agreement between the present LES and Kawamura et. al. DNS. The temperature fluctuations plotted on Fig. 5 are however underestimated except close to the wall. The biggest discrepancy is reached at the fluctuation peak where the LES captured fluctuations are 12\% lower than the DNS profile. Any following optical effect of the turbulent temperature field (see section 3.2) is therefore expected to be slightly underestimated as well.

\subsection{Optical calculations in the fluid}

The propagation of light is described as an electromagnetic wave following the Maxwell equations, where the medium is non-conducting and with a constant magnetic susceptibility. The speed of light being about 8 order of magnitude higher than the fluid velocity, the turbulence is considered as "frozen" from the laser beam point of view. The refractive index $n=\frac{c_{0}}{c}$, with $c$ the speed of light in the medium and $c_{0}$ the speed of light in free space, is taken constant in time. The propagation equation of the electric field $\mathbf{E}$ is then

$$
\nabla \mathbf{E}^{2}=\frac{n^{2}}{c_{0}^{2}} \frac{\partial^{2} \mathbf{E}}{\partial t^{2}}
$$

Adopting the classical approach used in aero-optics [13], the light field is modeled as a monochromatic sinusoidal plane wave propagating from $y=0$ to $y=L$. L is the distance traveled by the light wave. After the crossing of the full height of the channel, $L=2 h$. The assumption is here that the wavelength is small compared to the smallest turbulent structures and all diffraction effects are neglected. Equation. (6) then has a solution,

$$
\mathbf{E}(\mathbf{r}, t)=\mathbf{E}_{0} e^{i(\varphi(\mathbf{r}, k)-\omega t)},
$$

where $\mathbf{r}=(x, y, z)$ is the position, $t$ is the time, $\mathbf{E}_{0}$ is the constant field amplitude, $\varphi$ is the wave phase at $\mathbf{r}, k=\frac{2 \pi}{\lambda}$ is the wave number and $\omega$ is the wave frequency. The wave phase may vary as the laser beam crosses the turbulent medium, and it is written as the sum

$$
\varphi=\varphi_{0}+\Delta \varphi
$$

where $\varphi_{0}$ is the non-perturbed phase variation and $\Delta \varphi$ is the cumulative phase distortion from $y=0$ to $y=L . \Delta \varphi$ is related to the refractive index perturbation $\Delta n=n-n_{0}$ along the optical path, where $\Delta n$ is the difference to the refractive index in a non-perturbed medium $n_{0}$.

$$
\Delta \varphi=k \int_{0}^{L} \Delta n d y
$$

The wave number $k=\frac{2 \pi}{\lambda}$ depends on the monochromatic laser beam wavelength. In this work, the wavelength value 
was $\lambda=1.034 \times 10^{-6} \mathrm{~m}$, which is typical for Yb:YAG. As already mentioned, the refractive index in a gas varies with the density following the Gladstone-Dale law [8]. For the present flow calculation, the density varies at most by a few $10^{-3} \%$ with the pressure, and at most by a few $10 \%$ with the temperature. The variations with pressure are therefore neglected, and the refractive index depends only on the temperature, as

$$
n=1+G(\lambda) \times \rho(T)=1+\frac{0.044}{T},
$$

where $G=2.25 \times 10^{-4} \mathrm{~m}^{3} / \mathrm{kg}$ is the Gladstone-Dale constant.

To sum up, the laser beam wavefront deformation after crossing the turbulent channel can be computed from the LES instantaneous temperature field using equations 10 and 9. This method was applied to obtained the results presented below in section 3.2 and 3.3.

The Sutton model [13] links the phase distortion fluctuations to the density fluctuations. It originally assumes a homogeneous and isotropic turbulence, but it has been used in different flows including turbulent boundary layers by Wang et. al. [16]. The general form of the linking equation [31] is

$$
\Delta \varphi_{r m s}^{2}=G^{2} k^{2} \int_{0}^{L} \int_{0}^{L} R_{\rho^{\prime} \rho^{\prime}}\left(y, y^{\prime}\right) d y^{\prime} d y,
$$

where $R_{\rho^{\prime} \rho^{\prime}}$ is the two-point density correlation in the direction of the light propagation. The difficulty arises from attempted modelings of the right-hand term. The more widely used formulation of the Sutton model bases on the density correlation length $\Lambda$. It is equivalent to the previous formulation provided that $R_{\rho^{\prime} \rho^{\prime}}$ and $\Lambda$ are defined consistently [16].

$$
\Delta \varphi_{r m s}^{2}=2 G^{2} k^{2} \int_{0}^{L} \rho_{r m s}^{2}(y) \Lambda(y) d y
$$

The Sutton model alone does not allow to avoid conducting a Large Eddy Simulation. Indeed, the knowledge of the instantaneous densities is still needed in Eqn. (12) to calculate the variance of density fluctuations and the density correlation length. Yet it would be very interesting to be able to predict the wavefront deformation from mean quantities only, such as the one obtained with RANS simulations at a much cheaper numerical cost than LES. In order to investigate this possibility, a modeling of the density rms and the density correlation length was tested. The approach proposed by Tromeur et. al. [14] was here adapted to a thermal boundary layer. They assume $\Lambda$ and $\rho_{r m s}$ homogeneous over the direction of light propagation y and roughly approximate them. The density correlation length is modeled by $\Lambda=0.1 \delta$ where $\delta$ is the boundary layer thickness. The density rms is modeled by $\rho_{r m s}=A\left(\rho_{w}-\rho_{\infty}\right)$ where $\mathrm{A}$ is a parameter variable between 0.1 and $0.2, \rho_{w}$ is the wall density and

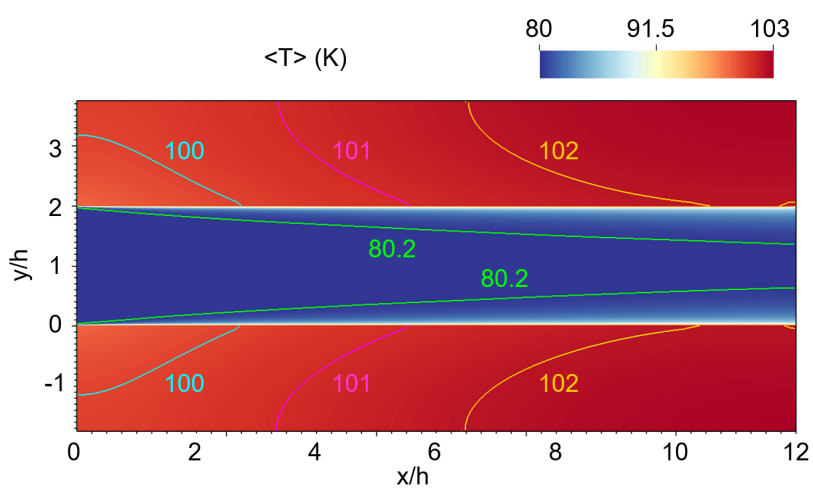

Fig. 6. Mean temperature on a streamwise plane in the channel flow, with contour line at constant temperature $T=80.2 \mathrm{~K}$, and in the two heated slabs, with contour lines at constant temperatures $T=100,101$ and $102 \mathrm{~K}$

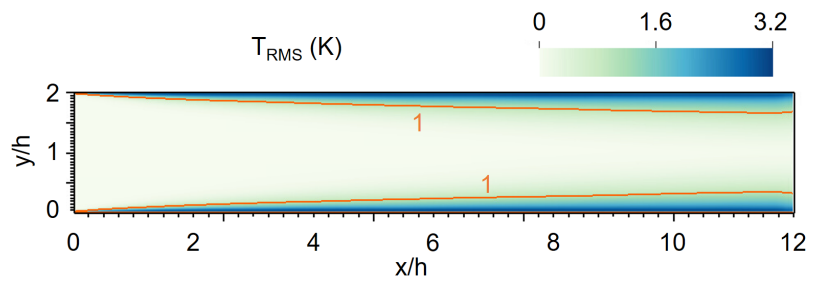

Fig. 7. Root mean square of temperature on a streamwise plane in the channel flow, with contour line at $T_{r m s}=1 \mathrm{~K}$

$\rho_{\infty}$ is the freestream density, here the centerline density. The modeled phase distortion variance is then given by

$$
\left(\Delta \varphi_{r m s}^{2}\right)_{m d l}=2 G^{2} k^{2} 0.1 \delta^{2} A^{2}\left(\rho_{w}-\rho_{\infty}\right)^{2}
$$

\section{Results and discussion}

\subsection{Temperatures in the open channel}

Figure 6 presents the mean temperature at thermal equilibrium on a $(x y)$ surface at $z / h=3$ (the spanwise middle of the simulated domain), in the open channel of helium (in blue) and in the two half slabs (in red). The helium flow enters the channel from the left side and it is heated up as it cools down the slabs while flowing to the right. The expected thermal boundary layers develop in the fluid along the flow beside the slabs. They are highlighted by the contour line at $T_{b l}=80.2 \mathrm{~K}$. The value of $T_{b l}$ is chosen so that $T_{w}-T_{b l}=99 \%\left(T_{w}-T_{f}\right)$ on average along the channel, where $T_{f}=80 \mathrm{~K}$ is the fluid temperature at the channel centerline and $T_{w}$ is the wall temperature. It is reminded that the flow is fully developed, and there is no viscous boundary layer. In the slabs, a temperature gradient is created between the entry corner near the fluid and the opposite corner. The heat transfer is higher at the beginning of the channel where the temperature gradients are stronger, than at the end of the channel where the boundary layer is thicker. The temperature difference between the two slab corners is $3 \mathrm{~K}$. The elongation coefficient for undoped crystal YAG at $100 \mathrm{~K}$ is 


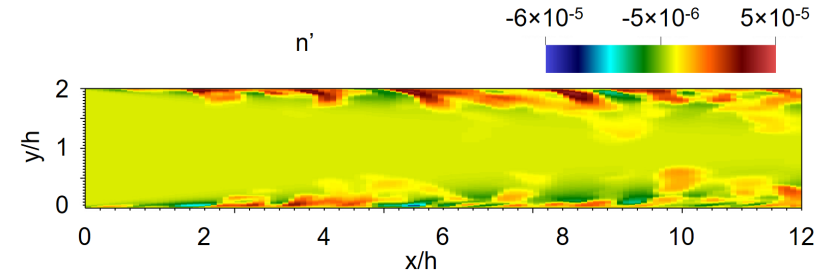

Fig. 8. Instantaneous refractive index fluctuations $n^{\prime}$, mapped on a streamwise plane in the channel flow

$\alpha=2 \times 10^{-6} \mathrm{~K}^{-1}$ [32], which corresponds to a small elongation: $\frac{\Delta L}{L}=6 \times 10^{-4} \%$ for $3 \mathrm{~K}$. The thermo-optic coefficient of $1 \%$-doped $\mathrm{Yb}$ :YAG at $100 \mathrm{~K}$ is $\frac{d n}{d T}=10^{-6} \mathrm{~K}^{-1}[10]$. Helium thermo-optic coefficient is not much bigger: it varies between $4.6 \times 10^{-6} \mathrm{~K}^{-1}$ at $100 \mathrm{~K}$ and $7.2 \times 10^{-6} \mathrm{~K}^{-1}$ at $80 \mathrm{~K}$ [9]. The fluid is however subjected to stronger temperature gradients compared to the solid. The maximum refractive index difference in the solid is $\Delta n_{s, \max }=2 \times 10^{-5}$ while it reaches $\Delta n_{f, \max }=1.2 \times 10^{-4}$ in the fluid.

Figure 7 presents the temperature root mean square (rms) at thermal equilibrium on a $(x y)$ surface $z / h=3$ in the open channel. The contour line at $T_{r m s}=1 \mathrm{~K}$ shows that the temperature fluctuates mostly in the thermal boundary layer, where the temperatures are higher. The peak of temperature fluctuations is situated close to the wall, where the velocity fluctuations are also the strongest. The temperature rms reaches there $3.2 \mathrm{~K}$. The temperature $\mathrm{rms}$ are not presented in the solid as they are in the range of $10^{-2} \mathrm{~K}$, which is small compared to the fluid. These small temperature fluctuations in the solid are consistent with its properties: Yb:YAG has a large thermal diffusivity $\alpha_{s}=\frac{\lambda_{s}}{\rho_{s} C_{p s}}=76 \mathrm{~mm}^{2} / \mathrm{s}$, when the fluid thermal diffusivity is $\alpha_{f}=4 \mathrm{~mm}^{2} / \mathrm{s}$.

\subsection{Optical outcome}

The refractive index is computed from the temperature field as detailed in Eqn. (10). All the usual turbulence statistics (mean, fluctuations, correlations...) can be computed for this quantity. Figure 8 is one example of refractive index fluctuations $n^{\prime}=n-\langle n\rangle$ on a $(x y)$ surface at $z / h=3$. It should be noted that $n^{\prime}$ differs from $\Delta n$, as used in Eqn. (9): the former is the difference to the mean refractive index, while the latter is the difference to the refractive index for an unperturbed medium. $\Delta n$ includes the perturbation due to the mean thermal boundary layer, whereas $n^{\prime}$ as plotted on Fig. 8 represents only the perturbation due to the temperature fluctuations. Figure 8 thus highlights the turbulence effect on the refractive index. Consistently with the dynamical and the thermal results, the refractive index fluctuations are located close to the walls, and their area widens toward the center of the channel with the thermal boundary layer development. The fluctuations display small values with peaks at $5 \times 10^{-5}$. By integrating a three-dimensional instantaneous refractive index field along the $y$ direction as written in the Eqn. (9), the wavefront deformation map presented in Fig. 9 was com-

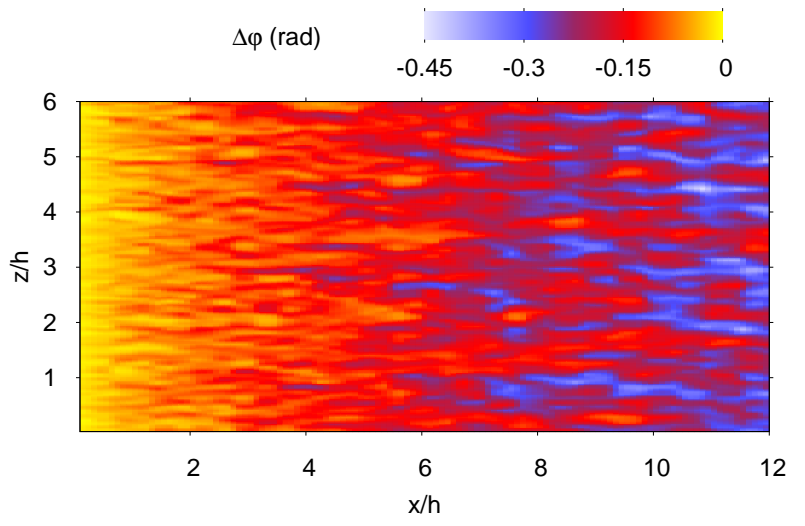

Fig. 9. Instantaneous wavefront deformation map on a spanwise plane after crossing the channel flow

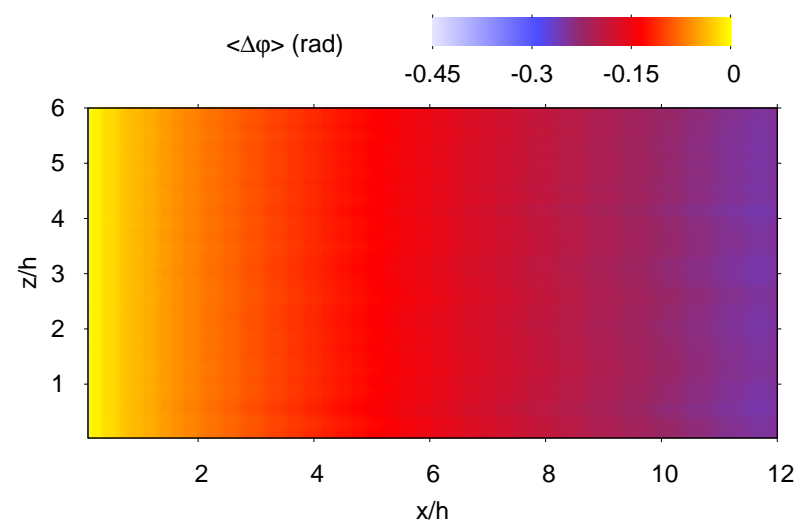

Fig. 10. Mean wavefront deformation map on a spanwise plane after crossing the channel flow

puted. In Fig. 9, $\Delta \varphi$ is the difference to the phase at $x / h=0$, where the fluid is uniformly at $80 \mathrm{~K}$. The fluctuations in the refractive index reflects on the instantaneous wavefront map, with fluctuations increasing along the channel. On average, the phase gets smaller along the channel, i.e. its absolute value increases, as the temperature increases and thus the refractive index decreases. This mean tendency is shown in Fig. 10. The mean wavefront deformation reaches $\langle\Delta \varphi\rangle=-0.24 \mathrm{rad}$ at $x / h=12$, the end of the simulated channel. The instantaneous wavefront deformation map presents higher values, up to $\Delta \varphi_{\max }=-0.45 \mathrm{rad}$ locally. A phase distortion could start to negatively impact the laser beam coherence from around $\Delta \varphi=\pi \mathrm{rad}$ as it corresponds to an optical path difference of half the beam wavefront $\lambda / 2$. It is however worth noting that this wavefront map represents only the effect of one cooling channel, and only about a quarter of it. In a typical multi-slabs amplifier, there could be about ten cooling channels crossed several times by the laser beam, which could then generate significant perturbations if the wavefront deformations caused by each channel add up.

Figure 11 presents the phase distortion rms after one crossing of the channel in the spanwise middle and at different streamwise positions. Consistently with the instantaneous 


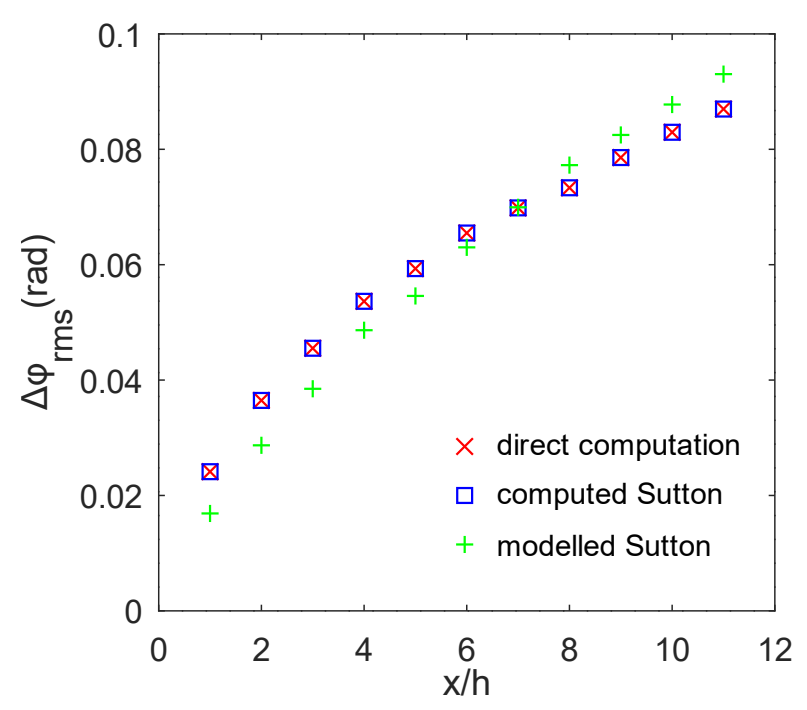

Fig. 11. Root mean square of phase distortion at $z / h=3$ and along the channel, calculated directly from the instantaneous phase distortions (Eqn. (9)), using the Sutton model with the instantaneous densities (Eqn. (11)) and using the Sutton model with the modelled density correlation length and rms (Eqn. (12) with $A=0.12$ )

data observed on Fig. 8 and 9, the phase distortion rms increases along the channel as the temperature field gets more turbulent, reaching $\Delta \varphi_{\mathrm{rms}}=0.9 \mathrm{rad}$ at the end of the simulated channel. It should be reminded here that the phase distortion rms is probably underestimated since the temperature rms were underestimated in the validation case (see section 2.2). The estimations of the phase distortion rms using the Sutton model are also plotted on Fig. 11. The general form of the linking equation (Eqn. (11)) when using the LES results yields excellent agreement with the direct computation after a complete crossing of the channel. Using the modeled density rms and correlation length (Eqn. (12)) allows a satisfactory agreement with the direct computation results. But this is only made possible by the adjustment of the variable $A$ of the model to this end. At this point, it is therefore hazardous to rely on the model alone.

\subsection{Effect of a stronger heating of the slabs}

To assess the effect of the optical heating of the solid on the laser beam, two additional simulations were conducted with heat sources three and six times higher than the heat source in the original simulation. The same procedure was adopted to attain the thermal equilibrium: the initial conditions were obtained from RANS calculations, and the solid heat capacity was reduced during the transient state. Figure 12 presents the mean temperatures for the three simulations. To compare them more conveniently, the temperatures for the three simulations are plotted on the same graph on a vertical profile close to the end of the channel $(x / h=11)$. The left flat part of the profiles corresponds to the temperatures in one half-slab while the right curved part corresponds to half of the channel, with the temperatures reaching $80 \mathrm{~K}$ for $y / h=1$ in the center of the channel. As expected, the

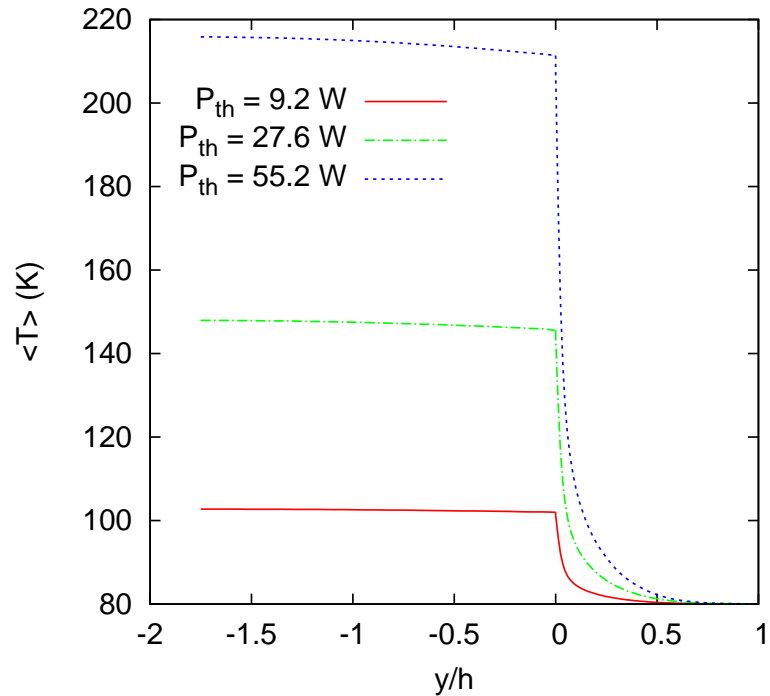

Fig. 12. Mean temperature on a vertical line in one half-slab and in half of the channel, at $x / h=11$ and at $z / h=3$, for different heat deposits in the slabs

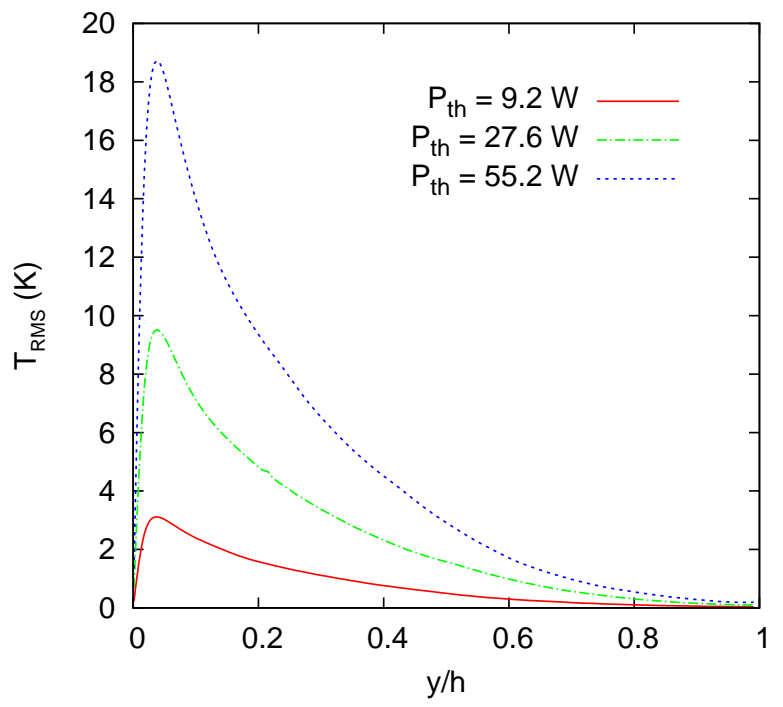

Fig. 13. Temperature root mean squares on a vertical line in half of the channel, at $x / h=11$ and at $z / h=3$, for different heat deposits in the slabs

slabs are hotter when the heating is stronger with an unchanged helium flow. Consequently, the temperature difference in the channel is increased: from $\Delta T=22 \mathrm{~K}$ between $y / h=0$ and $y / h=1$ at $x / h=11$ in the original simulation, it is tripled when the heat deposit is tripled, and multiplied par six, reaching $132 \mathrm{~K}$ at the same location, when the heat deposit is multiplied by six. This confirms that the equilibrium state was attained. Indeed, a simple thermal balance on the solid at thermal equilibrium shows that the solid to fluid heat transfer is then equal to the heat deposit. The solid/fluid temperature difference is thus expected to be proportional to the heat deposit at thermal equilibrium. The consequence for the temperature fluctuations are highlighted in Fig. 13 on which are plotted the temperature rms at the same location in 


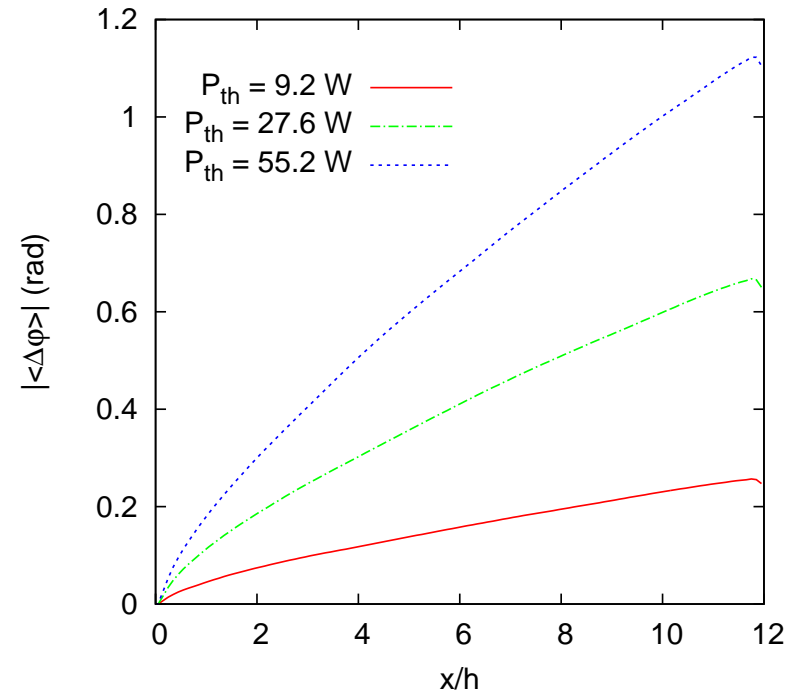

Fig. 14. Absolute value of the mean phase distortion at $z / h=3$ and along the channel for different heat deposits in the slabs

the half of the channel. The profile for a heat deposit multiplied by three or six collapses with the profile multiplied by respectively three or six of the original simulation.

Following the same procedure as for the original simulation, the refractive indexes were computed for the two additional simulations using Eqn. (10) and (9). Instantaneous and mean wavefront deformation maps similar to those plotted on Fig. 9 and 10 were obtained. To compare them more conveniently, they are plotted on profiles along the channel at $z / h=3$. Figure 14 presents such profile of the mean wavefront deformation. For the initial simulation, this profile is equivalent to a horizontal line in the $(x z)$ surface plotted on Fig. 10, except that the absolute value is plotted on Fig. 14. As expected, the absolute mean phase distortion is higher at stronger heating. Coherently with the Gladstone-Dale law, the mean phase rise is not however proportional with the heat deposit. At $x / h=11$ for instance, the mean phase distortion is 2.8 times higher at 3 times higher heat deposit, and 4.7 times higher at 6 times higher heat deposit. The mean phase distortion can be compensated by adaptive optics, unlike the phase distortion fluctuations.

Figure 15 presents the phase distortion rms for the three simulations. Again as expected, the phase distortion rms (plotted by lines) is higher at higher heat deposit but not proportionally. At $x / h=11$, the phase distortion rms is 2.6 times higher when the heat deposit is multiplied by 3 , and 4.3 times higher when it is multiplied by 6 . It is reminded here that these two additional simulations present a major limitation to their representativeness by lacking the increase of the fluid velocity that would accompany the increase of the slabs heating in a real case. Consequently, the increase of temperature fluctuations linked with a higher Reynolds number of the cooling flow is here overlooked.

For the sake of clarity, the Sutton model results based on LES instantaneous densities are not plotted on Fig. 15 as they collapse exactly with the direct computation results. But similarly to Fig. 11, the Sutton model results based on the mod-

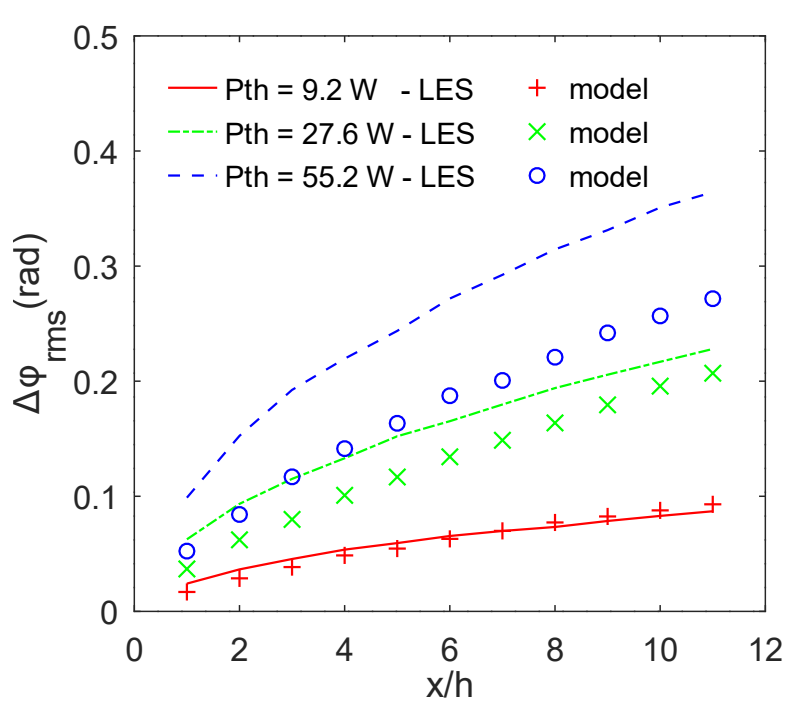

Fig. 15. Root mean square of phase distortion at $z / h=3$ and along the channel for different heat deposits in the slabs, calculated directly from the instantaneous phase distortions (Eqn. (9)) and using the Sutton model with the modeled density correlation length and $\mathrm{rms}$ (Eqn. (12) with $A=0.12$ )

eled density rms $\rho_{r m s}=A\left(\rho_{w}-\rho_{\infty}\right)$ and the modeled correlation length $\Lambda=0.1 \delta$ following Eqn. (12) are plotted with markers on Fig. 15. The same value $A=0.12$ was used for the three cases as it has allowed a satisfactory agreement with the directly computed results in the original case (see section 3.2). The model does not reproduce well the increase of phase distortion rms with the heating: it underestimates by $10 \%$ the phase distortion rms when the heat deposit is multiplied by 3 , and by up to $30 \%$ for the heat deposit multiplied by 6 . This outcome confirms the need to carry fine simulations such as LES to study the turbulence effect of a cooling flow on the laser beam. Indeed, even though the general form of the Sutton model has proven effective in this configuration, the need for the knowledge of the density fluctuations limits its applicability. The proposed modeling of the density rms as $\rho_{r m s}=A\left(\rho_{w}-\rho_{\infty}\right)$ does not lead to satisfactory results, and as long as an efficient alternative is not found, LES will remain mandatory for this application.

\section{Conclusion}

This work aimed at analyzing the effect of the turbulent cryogenic cooling of a laser amplifier on the output laser beam quality. To this end, Large Eddy Simulations of an open turbulent channel flow cooling two slabs at different optical heating were carried out. The phase distortions, mean and fluctuations, induced by an inhomogeneous and turbulent temperature field were investigated.

A moderate although non-negligible effect of the turbulence on the laser wavefront was found. It increases when the slabs heating increases. The phase distortion rms are of prime importance as they cannot be compensated by adaptive optics. Even though the Sutton model linking phase distortion rms to density rms was found theoretically accurate in this config- 
uration, its application using modeled density rms was not. Resort to Large Eddy Simulations thus remains necessary to investigate aero-optical effects in such laser amplifiers. Similar studies can be carried out for different amplifier designs and for different coolants. Liquid cryogenic fluids (e.g. nitrogen) are of special interest: although the cooling is more efficient than with gaseous helium, the refractive index varies more. It will then be unavoidable to pay attention to the induced phase distortion rms.

\section{Acknowledgements}

This work was granted access to the HPC resources of CINES under the allocations 2019-A0052A10626 and 2020A0072A10626 attributed by GENCI (Grand Équipement National de Calcul Intensif).

We acknowledge the financial support of the CrossDisciplinary Program on Numerical Simulation of CEA, the French Alternative Energies and Atomic Energy Commission.

\section{References}

[1] Wills, S., 2020. "ELI: Open for Business". Optics and Photonics News, 31(1), Jan., p. 30.

[2] Papadopoulos, D. N., Zou, J. P., Le Blanc, C., Ranc, L., Druon, F., Martin, L., Fréneaux, A., Beluze, A., Lebas, N., Chabanis, M., Bonnin, C., Accary, J. B., Garrec, B. L., Mathieu, F., and Audebert, P., 2019. "First commissioning results of the Apollon laser on the 1 PW beam line". In Conference on Lasers and Electro-Optics, OSA, p. STu3E.4.

[3] Le Garrec, B., 2010. "Laser-diode and Flash Lamp Pumped Solid-State Lasers". In AIP Conference Proceedings, Vol. 1228, AIP, pp. 111-116.

[4] Navratil, P., Slezak, O., Pilar, J., Ertel, K. G., Hanus, M., Banerjee, S., Phillips, P. J., Smith, J., De Vido, M., Lucianetti, A., Hernandez-Gomez, C., Edwards, C. B., Collier, J. L., Mocek, T., Mason, P. D., Divoký, M., and Butcher, T. J., 2018. "Characterization of Bivoj/DiPOLE 100: HiLASE 100-J/10-Hz diode pumped solid state laser". In Solid State Lasers XXVII: Technology and Devices, W. A. Clarkson and R. K. Shori, eds., SPIE, p. 33.

[5] Brown, D., Tornegård, S., Kolis, J., McMillen, C., Moore, C., Sanjeewa, L., and Hancock, C., 2016. "The Application of Cryogenic Laser Physics to the Development of High Average Power Ultra-Short Pulse Lasers". Applied Sciences, 6(1), Jan., p. 23.

[6] Brown, D. C., 2005. "The promise of cryogenic solidstate lasers". IEEE journal of selected topics in quantum electronics, 11(3), pp. 587-599.

[7] Mason, P. D., Fitton, M., Lintern, A., Banerjee, S., Ertel, K., Davenne, T., Hill, J., Blake, S. P., Phillips, P. J., Butcher, T. J., Smith, J. M., De Vido, M., Greenhalgh, R. J. S., Hernandez-Gomez, C., and Collier, J. L., 2015. "Scalable design for a high energy cryo- genic gas cooled diode pumped laser amplifier". Applied Optics, 54(13), May, p. 4227.

[8] Gladstone, J., and Dale, T., 1863. "XIV. Researches on the refraction, dispersion, and sensitiveness of liquids". Philosophical Transactions of the Royal Society of London, 153, Jan., pp. 317-343.

[9] Arp, V. D., McCarty, R. D., and Friend, D. G., 1998. Thermophysical Properties of Helium-4 from 0.8 to $1500 \mathrm{~K}$ with Pressures to $2000 \mathrm{MPa}$. Tech. rep., NIST, Sept.

[10] Cardinali, V., 2011. "Matériaux lasers dopés à l'ion ytterbium: Performances lasers en pompage par diodes lasers et étude des propriétés thermo-optiques à des températures cryogéniques”. $\mathrm{PhD}$ Thesis, Ecole Polytechnique $\mathrm{X}$.

[11] Roggemann, M. C., and Welsh, B., 1996. Imaging through Turbulence. The CRC Press Laser and Optical Science and Technology Series. CRC Press, Boca Raton.

[12] Gordeyev, S., Cress, J., and Jumper, E., 2013. "Far-field laser intensity drop-outs caused by turbulent boundary layers". Journal of Directed Energy, 5(1), pp. 58-75.

[13] Sutton, G., 1984. "Aero-optical foundations and applications". In 17th Fluid Dynamics, Plasma Dynamics, and Lasers Conference, American Institute of Aeronautics and Astronautics.

[14] Tromeur, E., Garnier, E., and Sagaut, P., 2006. “Analysis of the Sutton Model for Aero-Optical Properties of Compressible Boundary Layers". Journal of Fluids Engineering, 128(2), p. 239.

[15] Tromeur, E., Garnier, E., and Sagaut, P., 2006. "Largeeddy simulation of aero-optical effects in a spatially developing turbulent boundary layer". Journal of Turbulence, 7, Jan., p. N1.

[16] Wang, K., and Wang, M., 2012. "Aero-optics of subsonic turbulent boundary layers". Journal of Fluid Mechanics, 696, Apr., pp. 122-151.

[17] Truman, C. R., and Lee, M. J., 1990. "Effects of organized turbulence structures on the phase distortion in a coherent optical beam propagating through a turbulent shear flow". Physics of Fluids A: Fluid Dynamics, 2(5), May, pp. 851-857.

[18] Gordeyev, S., Cress, J. A., Smith, A., and Jumper, E. J., 2015. "Aero-optical measurements in a subsonic, turbulent boundary layer with non-adiabatic walls". Physics of Fluids, 27(4), Apr., p. 045110.

[19] Truman, C., 1992. "The influence of turbulent structure on optical phase distortion through turbulent shear flows". In Annual Interceptor Technology Conference, p. 2817.

[20] Angeli, P.-E., Bieder, U., and Fauchet, G., 2015. "Overview of the TrioCFD code: Main features, V\&V procedures and typical applications to nuclear engineering". In Proceedings of 16th International Topical Meeting on Nuclear Reactor Thermal Hydraulics (NURETH-16).

[21] Perin, J. P., Millet, F., Rus, B., and Divoký, M., 2011. "Cryogenic Cooling For High Power Laser Ampli- 
fiers". In 5th International Conference On The Frontiers Of Plasma Physics And Technology.

[22] Sagaut, P., 2002. Large Eddy Simulation for Incompressible Flows. Scientific Computation. Springer Berlin Heidelberg, Berlin, Heidelberg.

[23] Divoky, M., Sawicka, M., Sikocinski, P., Lucianetti, A., Novak, J., Rus, B., and Mocek, T., 2013. "Conceptual design of $100 \mathrm{~J}$ cryogenically-cooled multi-slab laser for fusion research". EPJ Web of Conferences, 59, p. 08004.

[24] Nicoud, F., and Ducros, F., 1999. "Subgrid-scale stress modelling based on the square of the velocity gradient tensor". Flow, turbulence and Combustion, 62(3), pp. 183-200.

[25] Calvin, C., Cueto, O., and Emonot, P., 2002. "An object-oriented approach to the design of fluid mechanics software". ESAIM: Mathematical Modelling and Numerical Analysis, 36(5), Sept., pp. 907-921.

[26] Mani, A., Wang, M., and Moin, P., 2008. "Resolution requirements for aero-optical simulations". Journal of Computational Physics, 227(21), Nov., pp. 9008-9020.

[27] Dittus, F. W., and Boelter, L. M. K., 1985. "Heat transfer in automobile radiators of the tubular type". International Communications in Heat and Mass Transfer, 12(1), pp. 3-22.

[28] Moser, R. D., Kim, J., and Mansour, N. N., 1999. "Direct numerical simulation of turbulent channel flow up to Re T=590". Physics of fluids, 11(4), pp. 943-945.

[29] Pope, S. B., 2000. Turbulent Flows, first ed. Cambridge University Press, Aug.

[30] Kawamura, H., Abe, H., and Matsuo, Y., 1999. "DNS of turbulent heat transfer in channel flow with respect to Reynolds and Prandtl number effects". International Journal of Heat and Fluid Flow, pp. 196-207.

[31] Havener, G., 1992. "Optical wave front variance A study on analytic models in use today". In 30th Aerospace Sciences Meeting and Exhibit, American Institute of Aeronautics and Astronautics.

[32] Aggarwal, R. L., Ripin, D. J., Ochoa, J. R., and Fan, T. Y., 2005. "Measurement of thermo-optic properties of Y3A15O12, Lu3Al5O12, YAIO3, LiYF4, LiLuF4,

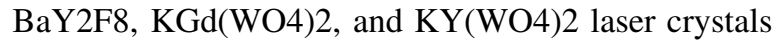
in the 80-300K temperature range". Journal of Applied Physics, 98(10), Nov., p. 103514. 